\title{
Properties of Gallium Lanthanum Sulphide Glass
}

\author{
Paul Bastock ${ }^{1}$, Chris Craig ${ }^{1}$, Khouler Khan ${ }^{1}$, Ed Weatherby $^{1}$, Jin Yao $^{2}$, Daniel William Hewak ${ }^{1}$ \\ ${ }^{I}$ Optoelectronics Research Centre, University of Southampton, Hampshire, SO17 1BJ \\ ${ }^{2}$ Department of Chemistry, University of Southampton, Hampshire, SO17 1BJ \\ p.bastock@soton.ac.uk
}

\begin{abstract}
A series of gallium lanthanum sulphide (GLS) glasses has been studied in order to ascertain properties across the entire glass forming region. This is the first comprehensive study of GLS glass over a wide compositional range.

OCIS codes: (260.3060) Infrared; (160.2290) Fiber materials
\end{abstract}

\section{Introduction}

GLS is a chalcogenide glass recognised for its valuable optical and thermal properties. It is used in many forms [1-3] and benefits active and passive mid-infrared applications requiring relatively low toxicity, high strength, large transparency window, high thermal stability, high solubility of rare earths, low thermal expansion and high laser damage threshold. Like all chalcogenide waveguides, current GLS fibre technology has not achieved the ultra-low losses achieved in silica based glasses. This is predominantly due to purity of commercial raw materials and the fabrication processes. Although chalcogenide optical fibres have been fabricated showing low but not ultra-low losses [4], these and fibres drawn from GLS glass have not achieved their predicted loss minima [5]. It is clear that continued development of both raw material purity and fabrication process is required. Here we present the first comprehensive optical and thermal characterisation of GLS carried out over a wide compositional range. Optical spectroscopy, ellipsometry and thermal analysis is used to determine glass transparency region, refractive index and thermal properties respectively. Determining relations between these parameters is key for device fabrication.

\section{Preparation of GLS glass samples}

Batches of homogeneously mixed $\mathrm{Ga}_{2} \mathrm{~S}_{3}$ and $\mathrm{La}_{2} \mathrm{~S}_{3}$ powders of various ratios (45:55- 75:25 mol\% $\mathrm{Ga}_{2} \mathrm{~S}_{3}$ : $\mathrm{mol} \% \mathrm{La}_{2} \mathrm{~S}_{3}$ ) were melted simultaneously for 24 hours in vitreous carbon crucibles at $1150{ }^{\circ} \mathrm{C}$ whilst under an argon purge. The purity of the as-received raw materials were 4 nines $(4 \mathrm{~N})$ and $3 \mathrm{~N}$, respectively, with respect to metallic impurities. It was found that heat treating the raw materials prior to melting significantly improved transmission. Figure 1 illustrates the improvement made in optical transparency through purification of raw materials. Following melting, the glasses were then annealed at $490{ }^{\circ} \mathrm{C}$. The 18 gram glass samples were individually cut into $15 \mathrm{~mm}$ diameter discs of various thicknesses and optically polished for characterisation as seen in Figure 2.

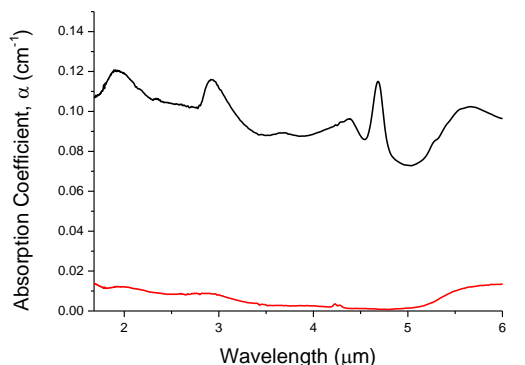

Figure 1: Effect of raw material purification on glass absorption using as-received raw materials (black) and heat treated raw materials (red)

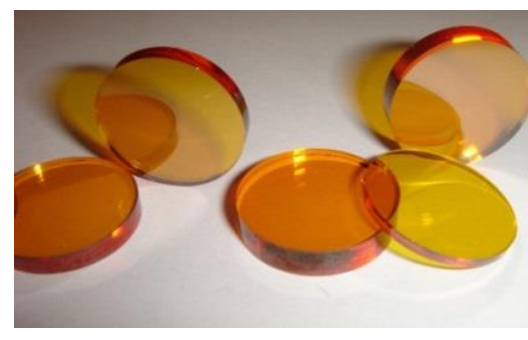

Figure 2: GLS glass discs polished for optical characterization

\section{Results}

Optical spectroscopy carried out on bulk samples was used to determine transparency regions from visible to infrared wavelengths. Reflection losses were taken into account by using identically polished thinner samples as reference in the dual beam UV-Vis-NIR, and as background in FTIR measurements. From the plots shown in Figure 3, it is quite evident that the 60:40 GLS has the widest transparency window, although all glasses are somewhat comparable. Glasses with a ratio of $45: 55 \mathrm{Ga}_{2} \mathrm{~S}_{3}$ to $\mathrm{La}_{2} \mathrm{~S}_{3}$ has a larger scattering due to phase separation, which was expected as it lies slightly outside the GLS glass forming region reported by Guittard et. al. [6]. Clear absorption peaks are seen at around $2.9,4.7$ and $6 \mu \mathrm{m}$ which correspond to $\mathrm{O}-\mathrm{H}, \mathrm{Ce}^{3+}$ ion and $\mathrm{H}_{2} \mathrm{O}$ absorption respectively. $\mathrm{Ce}^{3+}$ was inherently contained within the $\mathrm{La}_{2} \mathrm{~S}_{3}$ commercial raw material and was confirmed by glow discharge mass spectroscopy of the raw materials. The $\mathrm{O}-\mathrm{H}$ and $\mathrm{H}_{2} \mathrm{O}$ content was not intrinsic, and varied with melting conditions. 

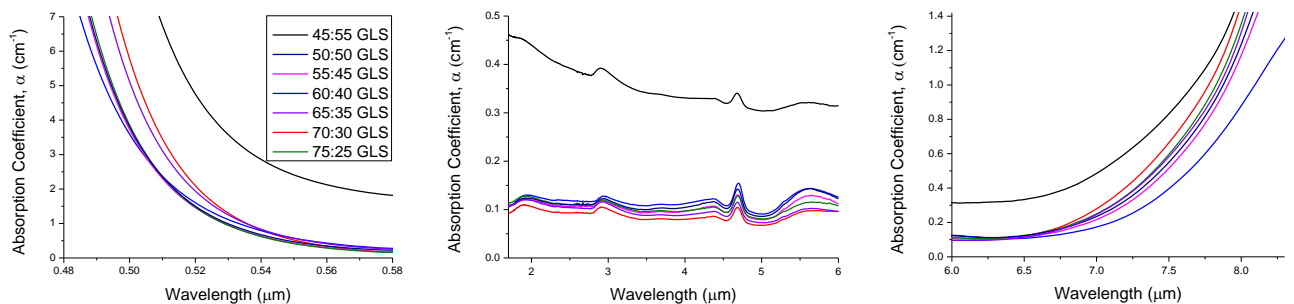

Figure 3: Optical transmission of GLS glass measured in region of (a) electronic absorption edge, (b) loss minimum and (c) infrared (multiphonon) absorption edge

The refractive index of the GLS was measured using an ellipsometer and found to decrease as a function of increasing wavelength, with 60:40 GLS bearing the highest refractive index of 2.51 at $0.65 \mu \mathrm{m}$ and 45:55 GLS possessing the lowest of 2.28, at $2.15 \mu \mathrm{m}$. Thermal properties of the GLS can express glass suitability for particular applications including fibre drawing. Table 1 lists the thermal properties of the GLS series, measured by differential thermal analysis, indicating thermal suitability for various applications.

Table 1: Thermal properties of GLS, $\left(\mathrm{T}_{\mathrm{g}}=\right.$ glass transition temperature, $\mathrm{T}_{\mathrm{x}}=$ onset crystallisation temperature, $\mathrm{T}_{\mathrm{p}}=$ peak crystallisation temperature, $\mathrm{T}_{\mathrm{m}}=$ onset melting temperature, $\mathrm{CTE}=$ coefficient of thermal expansion

\begin{tabular}{|c|c|c|c|c|c|}
\hline Glass Composition & $\mathbf{T}_{\mathbf{g}}\left({ }^{\circ} \mathbf{C}\right)$ & $\mathbf{T}_{\mathbf{x}}\left({ }^{\circ} \mathbf{C}\right)$ & $\mathbf{T}_{\mathbf{p}}\left({ }^{\circ} \mathbf{C}\right)$ & $\mathbf{T}_{\mathbf{m}}\left({ }^{\circ} \mathbf{C}\right)$ & $\mathbf{C T E}_{\left(\mathbf{2 0 - 3 0 0}{ }^{\circ} \mathbf{C}\right)} \mathbf{x 1 0} \mathbf{6}^{-\mathbf{6}}\left(\mathbf{K}^{-\mathbf{1}}\right)$ \\
\hline 45:55 GLS & 527.9 & 659.0 & 667.6 & 816.7 & 5.27 \\
\hline 50:50 GLS & 584.8 & 694.3 & 731.2 & 839.4 & 6.74 \\
\hline 55:45 GLS & 583.7 & 702.7 & 735.1 & 839.6 & 6.17 \\
\hline 60:40 GLS & 607.8 & 681.2 & 717.4 & 885.4 & 6.86 \\
\hline 65:35 GLS & 563.8 & 679.2 & 728.6 & 829.3 & 6.84 \\
\hline 70:30 GLS & 553.0 & 660.3 & 694.0 & 815.8 & 6.04 \\
\hline 75:25 GLS & 571.4 & 693.7 & 735.2 & 839.2 & 7.30 \\
\hline
\end{tabular}

It can be noted that 45:55 GLS and 70:30 GLS possess the lowest thermal onset temperatures, similarly to the refractive indices, which also show minima at the lower extreme of the glass formation range. Whereas 55:45 GLS bears the overall highest thermal onset temperatures and consistently high refractive index as a function of wavelength. The density of the GLS samples at room temperature follows the trend set by refractive index, as expected, where the lowest densities are attributable to the lowest refractive index.

\section{Discussion}

The results presented are intended to aid the matching of a glass stoichiometry to a particular application. For example in passive fibre applications, optical loss is critical. The results presented are dominated by extrinsic loss mechanisms but general trends between the refractive index and $\mathrm{T}_{\mathrm{g}}$ can be inferred; theoretically, fewer losses are expected in GLS with lower refractive index and $\mathrm{T}_{\mathrm{g}}$, within the glass formation range. Combining these results with thermal analysis information such as the Hrubý parameter and the CTE, the ideal compositions for core clad fibres that would achieve the lowest loss can be determined. It is hoped that as the glasses approach a dominant intrinsic loss mechanism, the relations between the theoretical and practical measurements can be linked. The $\mathrm{T}_{\mathrm{x}}$ allows selection for phase change memory applications whilst the $\mathrm{T}_{\mathrm{m}}$ gives insight for fibre drawing via the crucible drawing technique. The next step is to repeat this study with the lower loss materials currently being made as shown in Figure 1 and compare relationships.

\section{Conclusion}

The thermal and optical properties of bulk GLS glass has been measured and discussed for the entire glass forming stoichiometric range. Trends and relationships throughout the glass series has been summarised which can be used as reference when determining which GLS ratio is most suitable for a desired application.

\section{References}

1. Hewak, D.W., et al., Chalcogenide glasses for photonics device applications. GS Murugan, ed.(Research Signpost, Kerala, India, 2010) Chap, 2010. 2.

2. Youden, K., et al., Pulsed laser deposition of Ga-La-S chalcogenide glass thin film optical waveguides. Applied Physics Letters, 1993. 63(12): p. 1601-1603.

3. Mairaj, A., R. Curry, and D. Hewak, Chalcogenide glass thin films through inverted deposition and high velocity spinning. Electronics Letters, 2004. 40(7): p. 421-422.

4. Churbanov, M., et al., Optical fibers based on As-S-Se glass system. Journal of Non-Crystalline Solids, 2001. 284(1): p. 146-152.

5. Brady, D.J., et al., Minimum loss predictions and measurements in gallium lanthanum sulphide based glasses and fibre. Journal of Non-Crystalline Solids, 1998. 242(2-3): p. 92-98.

6. Guittard, M. and J. Flahaut, Rare earth sulfide and oxysulfide glasses, in New frontiers in rare earth science and applications. 1985. 